\title{
Characterization of CYP2B6 K262R Allelic Variants by Quantitative Allele-Specific Proteomics using a QconCAT Standard
}

DOI:

10.1016/j.jpba.2019.112901

\section{Document Version}

Accepted author manuscript

Link to publication record in Manchester Research Explorer

Citation for published version (APA):

Barber, J., Russell, M. R., Rostami-Hodjegan, A., \& Achour, B. (2020). Characterization of CYP2B6 K262R Allelic Variants by Quantitative Allele-Specific Proteomics using a QconCAT Standard. Journal of Pharmaceutical and Biomedical Analysis, 178, [112901]. https://doi.org/10.1016/j.jpba.2019.112901

\section{Published in:}

Journal of Pharmaceutical and Biomedical Analysis

\section{Citing this paper}

Please note that where the full-text provided on Manchester Research Explorer is the Author Accepted Manuscript or Proof version this may differ from the final Published version. If citing, it is advised that you check and use the publisher's definitive version.

\section{General rights}

Copyright and moral rights for the publications made accessible in the Research Explorer are retained by the authors and/or other copyright owners and it is a condition of accessing publications that users recognise and abide by the legal requirements associated with these rights.

\section{Takedown policy}

If you believe that this document breaches copyright please refer to the University of Manchester's Takedown Procedures [http://man.ac.uk/04Y6Bo] or contact uml.scholarlycommunications@manchester.ac.uk providing relevant details, so we can investigate your claim.

\section{OPEN ACCESS}




\section{Journal Pre-proof}

Characterization of CYP2B6 K262R allelic variants by quantitative allele-specific proteomics using a QconCAT standard

Jill Barber, Matthew R. Russell, Amin Rostami-Hodjegan, Brahim Achour

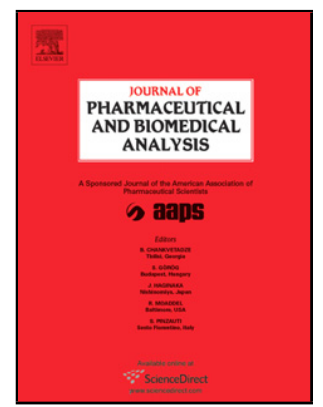

PII:

S0731-7085(19)31722-4

DOI: https://doi.org/10.1016/j.jpba.2019.112901

Reference: PBA 112901

To appear in: Journal of Pharmaceutical and Biomedical Analysis

Received Date: 26 July 2019

Revised Date: 12 September 2019

Accepted Date: 28 September 2019

Please cite this article as: Barber J, Russell MR, Rostami-Hodjegan A, Achour B, Characterization of CYP2B6 K262R allelic variants by quantitative allele-specific proteomics using a QconCAT standard, Journal of Pharmaceutical and Biomedical Analysis (2019), doi: https://doi.org/10.1016/j.jpba.2019.112901

This is a PDF file of an article that has undergone enhancements after acceptance, such as the addition of a cover page and metadata, and formatting for readability, but it is not yet the definitive version of record. This version will undergo additional copyediting, typesetting and review before it is published in its final form, but we are providing this version to give early visibility of the article. Please note that, during the production process, errors may be discovered which could affect the content, and all legal disclaimers that apply to the journal pertain.

(c) 2019 Published by Elsevier. 


\section{Characterization of CYP2B6 K262R Allelic Variants by Quantitative Allele-Specific Proteomics using a QconCAT Standard}

Jill Barber $^{a}$, Matthew R Russell ${ }^{b}$, Amin Rostami-Hodjegan ${ }^{a, c}$, Brahim Achour $^{a, *}$

${ }^{a}$ Centre for Applied Pharmacokinetic Research, University of Manchester, Stopford Building, Oxford Road, Manchester, M13 9PT, UK

${ }^{b}$ Stoller Biomarker Discovery Centre, University of Manchester, Nelson Street, Manchester, M13 9NQ, UK

${ }^{c}$ Certara UK Ltd. (Simcyp Division), 1 Concourse Way, Sheffield, S1 2BJ, UK

* Corresponding author at: Centre for Applied Pharmacokinetic Research, University of Manchester, Stopford Building, Oxford Road, UK, M13 9PT; Tel: +441613062375; Email: brahim.achour@manchester.ac.uk (B Achour) 


\section{Graphical Abstract}
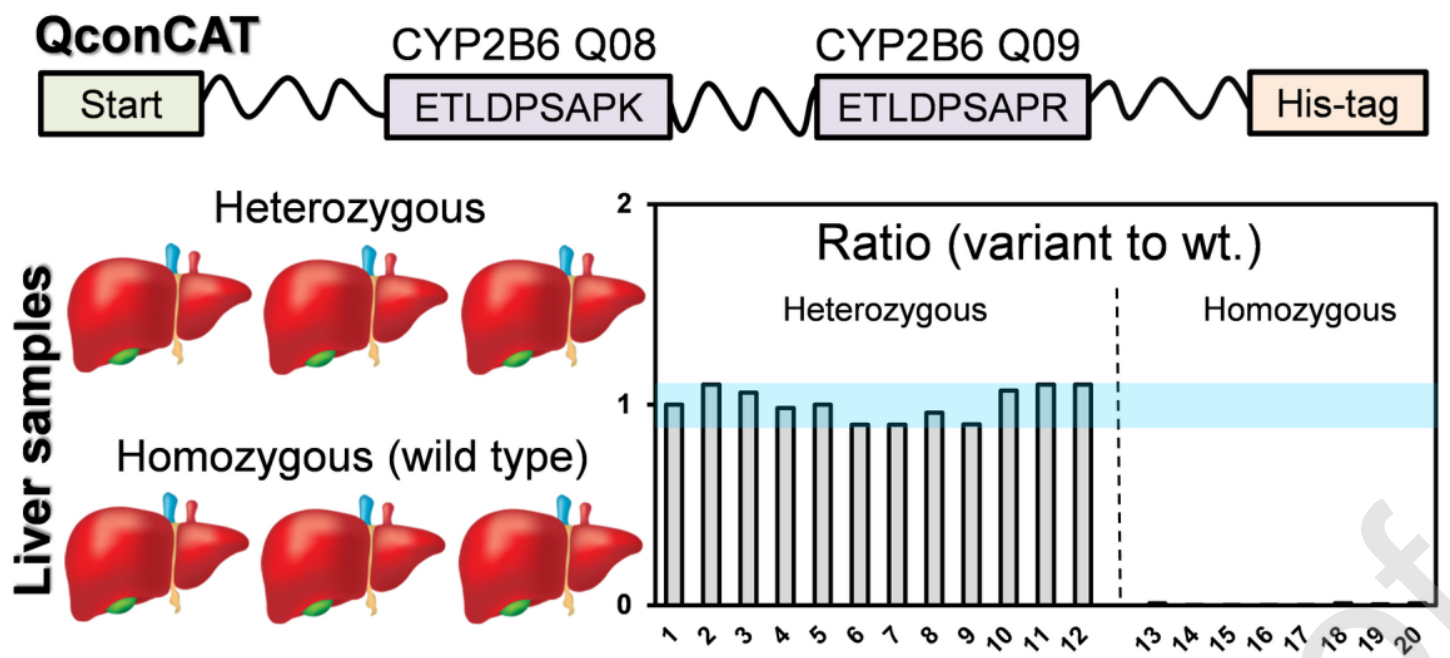

\section{Highlights}

- Development of QconCAT proteomic assay to characterize CYP2B6 K262R polymorphism

- Simultaneous phenotype determination and quantification of each allele product

- Assessment of correlation between CYP2B6 genotype and phenotype

- Assessment of correlation between allele-specific CYP2B6 levels and its activity 


\begin{abstract}
Clinically-relevant proteins are routinely characterized by targeted proteomic methods, which offer high accuracy and reproducibility. However, assays developed for these techniques lack distinction between different alleles expressed in biological samples. The significance of assessing such variations in genes relevant to pharmacology will depend on their prevalence and effects on drug therapy. We propose quantitative allele-specific proteomics for simultaneous abundance measurement and determination of missense polymorphisms. We employed a targeted proteomic strategy using a QconCAT standard which included two surrogate peptides (at 1:1 ratio) for a prevalent variation of CYP2B6 (K262R) so that the two variants could be quantified directly. Measurement was carried out in 24 human liver samples, out of which 21 were genotyped. Allele-specific analysis of CYP2B6 expression was accurate and precise $(\mathrm{CV}<9 \%)$, leading to determination of allele expression ratios (variant to wild type) for heterozygous $(1.006 \pm 0.079, \mathrm{n}=12)$ and homozygous $(0.005 \pm 0.004, \mathrm{n}=8)$ phenotypes. The abundance of CYP2B6 was $7.4 \pm 7.8 \mathrm{pmol} \mathrm{mg}^{-1}$ microsomal protein and showed good correlation with activity ( $\left.\mathrm{Rs}=0.91, \mathrm{p}<0.0001 ; \mathrm{R}^{2}=0.93\right)$. Comparable abundance (and activity) appeared to be associated with genotypes that express at least one wild type allele, which was corroborated by turnover values. This proof-of-principle study demonstrates the possibility of simultaneous determination of CYP2B6 phenotype and abundance by independent assessment of allele products.
\end{abstract}

\begin{abstract}
Abbreviations
CYP, cytochrome P450; HLM, human liver microsomes; LC, liquid chromatography; MRM, multiple reaction monitoring; MS, mass spectrometry; MS/MS, tandem mass spectrometry; QconCAT, quantification concatemer; wt, wild type.
\end{abstract}

\title{
Keywords
}

Proteomics; QconCAT; genotype; systems pharmacology; pharmacokinetics; human liver 


\section{Introduction}

Optimization of drug therapy using computational quantitative systems pharmacology models requires 'systems' information to be collected at different levels, including genotype and protein abundance for the target patients, and in vitro data for the drug against target proteins (e.g. enzymes, transporters and receptors) [1]. This highlights the potential role of 'omic' strategies, including quantitative proteomics, in informing and refining these models. A range of state-of-the-art proteomic approaches are currently available, with specific advantages and limitations, which determine their applications in pharmacology research [2]. Targeted proteomic quantification has traditionally relied on the use of standard peptides that are selected to allow quantification of the protein of interest, with one of the main criteria being avoidance of polymorphisms [3]. Advances in methodology allow us to turn the polymorphism challenge into an opportunity - we propose that clinically significant missense polymorphisms should be characterized to allow patient-to-patient variation in drug clearance to be better elucidated. A clinically significant polymorphism is arbitrarily defined as one that is prevalent $(>1 \%)$ in a certain population and is shown to affect activity [4].

Drug and xenobiotic metabolic clearance is mainly driven by the activity of cytochrome P450 (CYP) enzymes, which are encoded by 57 functional genes, out of which 12 isoforms belonging to three families are the main contributors to drug metabolism (CYP1A1, 1A2, 2A6, 2B6, 2C8, 2C9, 2C19, 2D6, 2E1, 2J2, 3A4 and 3A5). The CYP enzymes with the highest contribution to drug metabolism are hepatic CYP3A4/5, 2D6, 2C9, 1A2, 2B6 and 2C19, accounting for approximately $85 \%$ of drug metabolism pathways [5]. These enzymes have a highly polymorphic nature; some variants have similar activity to the wild type, but others are profoundly different. Therefore, identification of polymorphic variants of clinical significance and characterization of their protein products is required to improve drug pharmacokinetic predictions [6].

While the most widely studied cytochrome P450 enzymes for polymorphism are CYP2D6 and CYP2C9, the importance of CYP2B6 in drug metabolism has emerged recently owing to its inducibility, polymorphic nature and numerous substrates, including narrow therapeutic index drugs, such as the anti-cancer drug cyclophosphamide and the non-nucleoside reverse transcriptase inhibitor efavirenz, 
which is used against HIV [7]. A number of CYP2B6 polymorphisms with clinical consequences are highly prevalent in certain populations. For example, the variation K262R (CYP2B6*4, *6, *7, *13, $* 16, * 19, * 20, * 26, * 34, * 36, * 37$ and $* 38)$, which can lead to alterations in expression and catalytic activity, is particularly prevalent in South Asians, Africans and Caucasians [4].

We have previously described the principle of quantitative allele-specific proteomics (Q-ASP) of enzymes to characterize missense polymorphisms [8,9], however we did not provide details of the validation and application of this method. The aim of this study is therefore to describe the allelespecific assay and to demonstrate its application to CYP2B6 K262R variation. This application is intended to assess the possibility of simultaneously predicting the enzyme polymorphism (by determining the phenotype) and measuring its protein concentration using allele-specific proteomics in a well-characterized set of liver samples.

\section{Materials and Methods}

\subsection{Characteristics of human liver microsomal samples}

Individual human liver microsomal $(\mathrm{HLM}, \mathrm{n}=24)$ samples and one microsomal pool from 50 human livers were provided by Pfizer (Groton, CT) with associated demographic information, total cytochrome P450 content, genotype and enzyme activity data. The individual sample set included 23 liver microsomal samples from Caucasian subjects and one from an African American donor. The samples were acquired from BD Gentest or Vitron with ethical cover by the suppliers. Preparation of microsomes by the suppliers followed a standard protocol. CYP2B6 genotype information was available for 21 samples and 3 samples were not genotyped. Total cytochrome P450 was estimated for each individual sample using CO-difference spectroscopy. The pooled sample was used in this study for assay development. Table $\mathbf{S 1}$ shows characteristics of the sample donors.

\subsection{QconCAT standard}

The QconCAT standard (MetCAT), designed for the quantification of drug-metabolizing enzymes [8], included standard peptides for the quantification of 15 CYP enzymes in addition to peptides common to CYP sub-families. QconCAT expression and quality assessment is summarized in Supplementary 
Methods. The QconCAT sequence also included two allele-specific peptides representing CYP2B6 K262R polymorphism (Table 1).

\subsection{Sample preparation for proteomic analysis of CYP2B6}

Sample preparation for LC-MS analysis of CYP2B6 was performed as described previously $[8,9]$. Briefly, microsomal protein content in the samples was determined using the Bradford method. The amount of sample protein prepared was $26-46 \mu \mathrm{g}$ from individual microsomal samples using a gelbased method after solubilization in 5\% w/v SDS. Microsomal samples and isotope-labeled QconCAT standard (expressed in Escherichia coli) were diluted 1:10, loaded on 10\% w/v SDS-PAGE gels and analyzed in duplicate. Sample gel bands were excised between 45 and $65 \mathrm{kDa}$ (and $78 \mathrm{kDa}$ for the QconCAT). Protein disulfide bonds were reduced using dithiothreitol $\left(10 \mathrm{mM}, 50^{\circ} \mathrm{C}, 30 \mathrm{~min}\right)$ and alkylated with iodoacetamide (55 mM, room temperature, in the dark, 1 hour). Proteolysis was carried out in $25 \mathrm{mM}$ ammonium bicarbonate buffer, $\mathrm{pH} 8.0$, using lysyl endopeptidase $\left(1 \% \mathrm{w} / \mathrm{w}, 30^{\circ} \mathrm{C}, 4\right.$ hours) followed by trypsin $\left(1 \% \mathrm{w} / \mathrm{w}, 37^{\circ} \mathrm{C}\right.$, overnight $)$. Sample peptides were collected, spiked with QconCAT digest and transferred to MS vials for LC-MS analysis.

\subsection{Global proteomic analysis of CYP2B6}

Global proteomic analysis was performed to assess the feasibility of detecting the CYP2B6 peptide harboring the K262R polymorphism by LC-MS proteomics. Prepared liver digests (45 ng) from a subset of human liver microsomes $(n=4)$ were analyzed on a nanoACQUITY ${ }^{\text {TM }}$ UPLC ${ }^{\circledR}$ system (Waters, Manchester, UK) connected to a SYNAPT ${ }^{\mathrm{TM}}$ G2-Si mass spectrometer (Waters). Peptides were injected onto a Symmetry C18 trap column $(5 \mu \mathrm{m}, 180 \mu \mathrm{m} \times 20 \mathrm{~mm})$ and eluted onto a HSS T3 analytical column $(1.8 \mu \mathrm{m}, 75 \mu \mathrm{m} \times 250 \mathrm{~mm})$. The LC program consisted of a gradient ( 3 to $60 \%)$ of acetonitrile

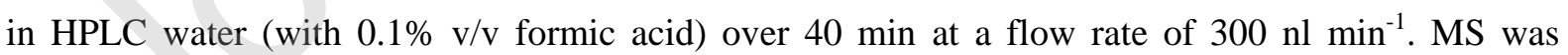
performed based on high-definition $\mathrm{MS}^{\mathrm{E}}$ [10]. Data analysis was done using MassLynx v4.1 and ProteinLynx Global Server (PLGS) v3.0.2 with IdentityE (Waters) search engine for data-independent acquisition. A list of all identified CYP2B6 peptides was then extracted. 


\subsection{Targeted allele-specific proteomic analysis of CYP2B6}

After confirming the detection of the polymorphic peptide (ETLDPSAPK) using global proteomics (Table S2), targeted allele-specific quantification was carried out to assess the levels of the two variants. The QconCAT sequence included heavy isotope-labeled versions of the two surrogate peptides (ETLDPSAPK, ETLDPSAPR). Microsomal material (0.6-1.3 $\mu \mathrm{g})$ from 24 liver samples was analyzed using scheduled multiple reaction monitoring (MRM) on a nanoACQUITY nano-HPLC system (Waters, UK) coupled to a TSQ Vantage triple quadrupole mass spectrometer (Thermo Fisher Scientific, USA) operated using Xcalibur v2.0.6 (Thermo Fisher). Peptides were eluted from a Waters HSS T3 C18 analytical column $(1.8 \mu \mathrm{m}, 75 \mu \mathrm{m} \times 150 \mathrm{~mm})$ at a flow rate of $300 \mathrm{nl} \mathrm{min}^{-1}$ with a gradient of $3 \%$ to $50 \%$ acetonitrile over $40 \mathrm{~min}$. Skyline v1.4 was used to design the transitions (Table 1) and Skyline v4.2 was used to analyze the data. Samples were interspersed by blanks, which were analyzed to assess carry-over. For quantitative assay development, a pooled sample from 50 livers $(30 \mu \mathrm{g})$ was prepared and analyzed using the designed assay to assess technical, analytical, intra-day and inter-day variability. Linearity was evaluated by analyzing a range of $0.08-2.31 \mu \mathrm{g}$ of microsomal pool protein.

\subsection{Targeted proteomic data analysis}

Data analysis followed our previously published methodology [9] with additional considerations for the purity of the QconCAT standard. Skyline v4.2 was used for analysis of MRM data. Quantitative ratios were calculated based on signal intensity (area ratios) of three fragment ions from each peptide. Allelespecific measurement of CYP2B6 was performed by measuring surrogate peptides for each allele separately (ETLDPSAPK for wild type, ETLDPSAPR for variant). For example, to characterize CYP2B6 K262R in liver sample 1 (genotyped as $* 1 / * 6$ ), the abundance of CYP2B6 was measured using Equation 1 and the ratio of variant to wild type was determined according to Equation 2.

$$
\begin{aligned}
& {[\mathrm{CYP} 2 \mathrm{~B} 6]=[\mathrm{CYP} 2 \mathrm{~B} 6 * 1]+[\mathrm{CYP} 2 \mathrm{~B} 6 * 6]=[\text { ETLDPSAPK }]+[\text { ETLDPSAPR }]} \\
& R_{\text {variant } / w t}=[\mathrm{CYP} 2 \mathrm{~B} 6 * 6] /[\mathrm{CYP} 2 \mathrm{~B} 6 * 1]=[\text { ETLDPSAPR }] /[\text { ETLDPSAPK }]
\end{aligned}
$$

The terms [ETLDPSAPK] and [ETLDPSAPR] refer to the concentration of the two variant peptides in each liver sample measured using LC-MS/MS as previously described [9]. 


\subsection{Assessment of catalytic activity and drug turnover}

CYP2B6 catalytic activity rates were determined as previously described [11]. Briefly, the metabolite of mephenytoin (N-desmethyl mephenytoin) was monitored using HPLC-SRM MS/MS. The internal standard used was the deuterated version of the metabolite. Vmax and abundance data were used to calculate mephenytoin turnover $\left(\mathrm{k}_{\mathrm{cat}}\right)$ as follows:

$$
k_{\mathrm{cat}}=\mathrm{V}_{\mathrm{max}} /[\mathrm{CYP} 2 \mathrm{~B} 6]
$$

\subsection{Statistical data analysis}

All statistical data analyses were performed using Microsoft Excel 2010, GraphPad Prism® v7.04 (GraphPad Software, La Jolla, California, USA) and R v3.5.2. Spearman rank-order correlation (Rs) test was used to assess abundance-activity correlation, and the level of scatter was evaluated by linear regression $\left(\mathrm{R}^{2}\right)$. Analysis of variance (ANOVA) was used to assess differences between genotype groups. The $p$-value cut-off was set at $\alpha=0.05$ and Bonferroni-corrected $\left(\alpha^{\prime}=0.017\right)$ for iterative posthoc analyses using Tukey-Kramer test (four sub-sets with $\geq 3$ samples). Principal components analysis (PCA) was used for multivariate analysis to differentiate sources of variability in abundance.

\section{Results}

\subsection{Quality assessment of allele-specific proteomic measurement}

Quality assessment of the expressed QconCAT (MetCAT) standard is shown in Supplementary Results. Global proteomic data showed the feasibility of detecting the polymorphic peptide in human liver samples (Table S2). To assess the quality of targeted proteomic quantification using the QconCAT-MRM assay, quality control measurements were carried out on a pooled sample of 50 livers. These showed analytical variability (estimated as \%CV) within $4 \%$ for both CYP2B6 peptides (<3\% for the wild type peptide, ETLDPSAPK, and $<4 \%$ for the variant peptide, ETLDPSAPR). Technical variability was higher (7-8\%) for the two peptides (Fig. 1A). Intra-day and inter-day technical variability was assessed as within $9 \%$ for both peptides $(<3 \%$ and $<9 \%$, respectively, for ETLDPSAPK, and $<4 \%$ and $<9 \%$, respectively, for ETLDPSAPR) (Fig. 1B). Linearity was demonstrated for the two peptides over a practical range $(0.07-2.31 \mu \mathrm{g})$ of microsomal protein $($ Fig. 1C). This translated to a measured peptide range of 0.63-21.20 fmol of ETLDPSAPK and 0.28-7.20 fmol of ETLDPSAPR. The 
amount of protein analyzed from the 24 individual samples was in the range $0.60-1.30 \mu \mathrm{g}$ and the measured peptide was in the range 0.75-10.84 fmol and 0.31-6.51 fmol of ETLDPSAPK and ETLDPSAPR, respectively. This analysis confirms precision and linearity of the assay for the purpose of the quantification of CYP2B6 in the 24 individual samples.

\subsection{Phenotype determination from allele-specific proteomic data}

The two variant peptides were analyzed in each individual sample and were readily distinguishable in the MS/MS data. Phenotype characterization was therefore possible and was in all cases in agreement with available genotype data $(n=21)$ (Fig. 2). Subjects could be classified to "heterozygous", "homozygous (wild type)", and "homozygous (variant)" for the particular polymorphism (K262R). This assessment could be done based on the LC-MS/MS signal independently from abundance measurement. Fig. 2A shows examples of allele-specific analysis and Fig. 2B shows assessment of allele expression in genotyped samples $(\mathrm{n}=21)$. For heterozygous livers, the percentage of the abundance of the variant allele (relative to total CYP2B6) was consistently approximately $50 \%(50.25 \% \pm 1.66 \%$, range $48 \%$ $52 \%$ ) or expressed as a ratio of variant to wild type allele: $1.006 \pm 0.079$. For homozygous (wild type) livers, the percentage was almost zero: $0.48 \% \pm 0.45 \%$, range $0.1 \%-1 \%$ (variant to wild type ratio: $0.005 \pm 0.004$ ), indicating the absence of the variant allele product. This is shown in Fig. $2 \mathbf{B}$ and

Table 2. One additional liver (Sample 21) was also genotyped (homozygous for the variant *6); the percentage of variant to total CYP2B6 was approximately 100\% (Table 2). Three livers were of unknown genotype, and we therefore attempted to predict the genotype from the allele-specific proteomic data. For two livers (Livers 22 and 23, Table 2), the wild type peptide appeared at 50 times the concentration of the variant (percentage of variant to total CYP2B6 at approximately 2\%), suggesting that these samples were homozygous for wild type. By contrast, the remaining sample (liver 24) showed a 1:1 ratio of the two forms, indicating heterozygosity.

\subsection{Abundance-activity correlation and the effect of genotype}

Q-ASP measurement of CYP2B6 was performed based on the concentration of the two variant peptides

(Fig. 3A and B). Correlation with activity was assessed, reflecting a strong and significant abundanceactivity relationship (Fig. 3C). The strong correlation between abundance and activity in this genetically heterogeneous set of samples is in line with the very similar mephenytoin $\mathrm{k}_{\text {cat }}$ values for homozygous 
wild type and heterozygous donors, $5.6 \pm 2.0 \mathrm{~min}^{-1}$ and $6.4 \pm 2.3 \mathrm{~min}^{-1}$, respectively (Fig. 3D). The $\mathrm{k}_{\text {cat }}$ value for the homozygous $* 6$ sample was $21.1 \mathrm{~min}^{-1}$, representing a higher drug turnover than wild type and heterozygous samples. Differences in protein expression and activity levels between genotype groups were assessed using one-way ANOVA, returning no statistically significant difference (four groups with $n \geq 3$ ), possibly due to low sample numbers in several variant sub-sets (Table 3). This was confirmed by post-hoc statistical analysis (Tukey-Kramer test). Notably, the abundance levels in each genotype sub-set were in agreement with measured activity data, reflecting a consistent trend of variation in activity and abundance (mirror-image) across genotype groups (Fig. $\mathbf{3 E}$ ).

\subsection{Inter-individual variability in allele-specific expression of CYP2B6}

The measured abundance of CYP2B6 was $7.4 \pm 7.8 \mathrm{pmol} \mathrm{mg}^{-1}$ microsomal protein (Table S3), expressed as mean $\pm \mathrm{SD}(\mathrm{CV}=105 \%$, fold difference $=40.5)$, representing an average of $3 \%$ of the total CYP content in these samples (Fig. 3B). The measured technical variability $(\mathrm{CV}$ of $<9 \%)$ indicates that out of the 40-fold variation in measured abundance of CYP2B6 in the 24 donors, 1.4 fold is estimated to be due to technical error $\left(95^{\text {th }}\right.$ percentile $/ 5^{\text {th }}$ percentile $=$ $\left.\left[1+2 \times \mathrm{CV}_{\text {technical }}\right] /\left[1-2 \times \mathrm{CV}_{\text {technical }}\right]\right)$, and approximately 30 fold is assumed to reflect biological factors, including genetic variation. A 2-fold difference in mean values (of groups with $\geq 3$ samples) was observed between different genotype sub-sets (Fig. 3E, Table 3). Comparison between abundance and activity levels within genotype sub-sets revealed similar abundance and activity between genotypes that have at least one wild type allele (Fig. 3F). Principal components analysis confirmed higher variability $(79.6 \%)$ within than across genotype groups $(19.3 \%)$. Considering other co-variates of expression, there was little difference in CYP2B6 abundance between male $(n=14)$ and female $(n=10)$ donors $(p>0.05)$ and only a weak correlation with age $\left(R s=-0.09, p>0.05 ; R^{2}=0.02\right)$. Most donors were either overweight $(n=11)$ or obese $(n=8)$, with very little difference in abundance between body mass index (BMI) sub-sets.

\section{Discussion}

The use of in vitro data and pharmacokinetic modeling has led to improved predictions of the metabolic fate of drugs within the body by scaling to in vivo consequences. The collection of physiological data 
using a variety of 'omic' methods is expected to facilitate such predictions, leading to better tailored dosing in patient populations [1,6]. However, the potential of different methods (i.e. genomics, transcriptomics, proteomics and metabolomics) to provide certain types of information has yet to be fully explored. The aim of the current study was to assess the possibility of predicting a prevalent CYP2B6 missense polymorphism (K262R) and measuring CYP2B6 abundance simultaneously using allele-specific proteomics in a well-characterized set of liver samples. Although conventional genotyping can be carried out less invasively in blood samples, genotyping does not provide the required information about expression levels of enzymes in liver (the main site of drug metabolism). Consequently, the development of this assay has the potential to extend the utility of quantitative proteomics to determining the enzyme phenotype as well as expression levels, allowing better and more comprehensive characterization in the same assay.

The QconCAT method is arguably the best suited approach for allele-specific quantification because of the strict 1:1 stoichiometry of the standard, allowed by concatenation of peptides representing allelic variants, leading to accurate assessment of the relative contribution of these variants to total protein expression [12]. The sequence of the QconCAT designed in our laboratory for the quantification of enzymes (MetCAT) [8] contained variant peptides for CYP2B6, intended to target a prevalent genetic variation. A different application of this technique was demonstrated recently for hepatic UGT2B15, in which elucidation of the mechanism of genetic regulation of expression was carried out [13].

Using the proposed technique, the ratios of variant to wild type CYP2B6 levels reflected the genotype with minimal technical variability, demonstrating high precision and accuracy, in line with FDA guidelines for bioanalytical method validation. The measured abundance and variability of CYP2B6 were in agreement with previous studies using targeted and global proteomics [14,15]. CYP2B6 abundance was shown to represent on average 3\% of the total cytochrome P450 content in the sample set, in line with recent meta-analysis data [16]. The effects of demographic factors, including age and sex, on CYP2B6 abundance were not significant, which corroborates published findings [17].

A strong and significant abundance-activity correlation was demonstrated for CYP2B6 in the set of 24 livers. Correlation between abundance and enzymatic activity is routinely used to confirm the validity of proteomic quantification of enzymes in various laboratories. Ultimately, activity is associated with 
metabolic clearance, and therefore, protein levels should reflect activity rates in order to be used for pharmacokinetic predictions. Comparison of abundance and activity levels in genotype groups showed overall agreement; the mirror-image trend in Fig. 3E reinforces the conclusion that the different variants of CYP2B6 under study are of comparable catalytic activity $\left(\mathrm{k}_{\mathrm{cat}}\right)$, and that variations in activity rates are almost entirely due to differences in protein expression. Strictly, there were insufficient data to demonstrate this observation in the case of homozygous variant genotypes (the single $* 6 / * 6$ sample showed a higher rate of catalysis), but this trend holds for homozygous wild type and heterozygous expression.

These findings are largely supported by reports of comparable activity in donors expressing wild type CYP2B6 and those with heterozygous genotype $(* 1 / * 6)$ against bupropion and efavirenz [18]. However, differences in activity were reported for CYP2B6*6 homozygous variant against various CYP2B6 substrates and these differences tended to be substrate specific $[18,19]$. In a large scale study [17], higher activity was also demonstrated for both substrates with samples expressing homozygous wild type allele compared to $* 1 / * 6$ and $* 6 / * 6$ (rank order: $* 1 / * 1>* 1 / * 6>* 6 / * 6$ ); activity rates of $* 1 / * 2$ and $* 1 / * 5$ samples were comparable to $* 1 / * 6$. A rigorous investigation of CYP2B 6 activity for different genotypes against various substrates is therefore warranted but beyond the scope of this study. In line with abundance data, $\mathrm{k}_{\mathrm{cat}}$ values for mephenytoin were very similar for homozygous wild type and heterozygous samples; however, the turnover number in the single homozygous variant was higher. Higher $\mathrm{k}_{\text {cat }}$ for the CYP2B $6 * 6 / * 6$ donor could be due to its very low abundance in this sample but this remain speculative. Previous assessment of $\mathrm{k}_{\text {cat }}$ for bupropion, 7-ethoxy-4-trifluoro-methylcoumarin and efavirenz in a CYP2B6-overexpressed system [19] showed higher turnover by the *6 variant relative to wild type.

\section{Conclusion}

In this study, we present a case for the application of allele-specific proteomics to simultaneously measure enzyme abundance and determine missense polymorphisms in a well-defined set of samples. This information correlated well with activity and should therefore be applicable to prediction of drug 
clearance. Similar methodology is applicable to the characterization of a range of prevalent polymorphisms in drug-metabolizing CYP enzymes (Table S4).

\section{Conflict of interest}

The authors declare no conflict of interest.

\section{Acknowledgements}

The authors thank Pfizer (Groton, CT) for providing the HLM samples with associated data.

\section{Funding}

This research did not receive any specific grant from funding agencies in the public, commercial, or not-for-profit sectors.

\section{References}

[1] A. Rostami-Hodjegan, Physiologically based pharmacokinetics joined with in vitro-in vivo extrapolation of ADME: A marriage under the arch of systems pharmacology, Clin. Pharmacol. Ther., 92 (2012) 50-61. https://doi.org/10.1038/clpt.2012.65.

[2] B. Prasad, B. Achour, P. Artursson, C.E.C.A. Hop, Y. Lai, P.C. Smith, J. Barber, J.R. Wisniewski, D. Spellman, Y. Uchida, M.A. Zientek, J.D. Unadkat, A. Rostami-Hodjegan, Toward a consensus on applying quantitative LC-MS/MS proteomics in translational pharmacology research: A white paper, Clin. Pharmacol. Ther., (2019) [in press]. https://doi.org/10.1002/cpt.1537

[3] J. Kamiie, S. Ohtsuki, R. Iwase, K. Ohmine, Y. Katsukura, K. Yanai, Y. Sekine, Y. Uchida, S. Ito, T. Terasaki, Quantitative Atlas of membrane transporter proteins: Development and application of a highly sensitive simultaneous LC/MS/MS method combined with novel in-silico peptide selection criteria, Pharm. Res., 25 (2008) 1469-1483. https://doi.org/10.1007/s11095-008-9532-4

[4] Y. Zhou, M. Ingelman-Sundberg, V.M. Lauschke, Worldwide distribution of cytochrome P450 alleles: A meta-analysis of population-scale sequencing projects. Clin. Pharmacol. Ther., 102 (2017) 688-700. https://doi.org/10.1002/cpt.690

[5] U.M. Zanger, K. Klein, M. Thomas, J.K. Rieger, R. Tremmel, B.A. Kandel, M. Klein, T. Magdy, Genetics, epigenetics, and regulation of drug-metabolizing cytochrome P450 enzymes, Clin. Pharmacol. Ther., 95 (2014) 258-261. https://doi.org/10.1038/clpt.2013.220

[6] T.M. Polasek, S. Shakib, A. Rostami-Hodjegan, Precision dosing in clinical medicine: Present and future, Expert Rev. Clin. Pharmacol., $11 \quad$ (2018) 743-746. https://doi.org/10.1080/17512433.2018.1501271

[7] H. Wang, L.M. Tompkins, CYP2B6: New insights into a historically overlooked cytochrome P450 isozyme, Curr. Drug Metab., 9 (2008) 589-610. https://doi.org/10.2174/138920008785821710 
[8] M.R. Russell, B. Achour, E.A. McKenzie, R. Lopez, M.D. Harwood, A. Rostami-Hodjegan, J. Barber, Alternative fusion protein strategies to express recalcitrant QconCAT proteins for quantitative proteomics of human drug metabolizing enzymes and transporters, J. Proteome Res., 12 (2013) 5934-5942. https://doi.org/10.1021/pr400279u

[9] B. Achour, M.R. Russell, J. Barber, A. Rostami-Hodjegan, Simultaneous quantification of the abundance of several cytochrome $\mathrm{P} 450$ and uridine 5'-diphospho-glucuronosyltransferase enzymes in human liver microsomes using multiplexed targeted proteomics, Drug Metab. Dispos., 42 (2014) 500-510. https://doi.org/10.1124/dmd.113.055632

[10] B. Achour, H. Al Feteisi, F. Lanucara, A. Rostami-Hodjegan, J. Barber, Global proteomic analysis of human liver microsomes: Rapid characterization and quantification of hepatic drug-metabolizing enzymes, Drug Metab. Dispos., 45 (2017) 666-675. https://doi.org/10.1124/dmd.116.074732

[11] R.L. Walsky, R.S. Obach, Validated assays for human cytochrome P450 activities, Drug Metab. Dispos., 32 (2004) 647-660. https://doi.org/10.1124/dmd.32.6.647

[12] B. Achour, Z.M. Al-Majdoub, H. Al Feteisi, Y. Elmorsi, A. Rostami-Hodjegan, J. Barber, Ten years of QconCATs: Application of multiplexed quantification to small medically relevant proteomes. Int. J. Mass Spectrom., 391 (2015) 93-104. https://doi.org/10.1016/j.ijms.2015.08.003

[13] J. Shi, X. Wang, H. Zhu, H. Jiang, D. Wang, A. Nesvizhskii, H.-J. Zhu, Determining allele-specific protein expression (ASPE) using a novel quantitative concatamer based proteomics method, J. Proteome Res., 17 (2018) 3606-3612. https://doi.org/10.1021/acs.jproteome.8b00620

[14] S. Ohtsuki, O. Schaefer, H. Kawakami, T. Inoue, S. Liehner, A. Saito, N. Ishiguro, W. Kishimoto, E. Ludwig-Schwellinger, T. Ebner, T. Terasaki, Simultaneous absolute protein quantification of transporters, cytochrome P450s and UDP-glucuronosyltransferases as a novel approach for the characterization of individual human liver: Comparison with mRNA levels and activities. Drug Metab. Dispos., 40 (2012) 83-92. https://doi.org/10.1124/dmd.111.042259

[15] N. Couto, Z.M. Al-Majdoub, B. Achour, P.C. Wright, A. Rostami-Hodjegan, J. Barber, Quantification of proteins involved in drug metabolism and disposition in the human liver using label-free global proteomics. Mol. Pharm., $16 \quad$ (2019) 632-647. https://doi.org/10.1021/acs.molpharmaceut.8b00941

[16] B. Achour, J. Barber, A. Rostami-Hodjegan, Expression of hepatic drug-metabolizing cytochrome P450 enzymes and their intercorrelations: A meta-analysis. Drug. Metab. Dispos., 42 (2014) 13491356. https://doi.org/10.1124/dmd.114.058834

[17] Z. Desta, T. Saussele, B. Ward, J. Blievernicht, L. Li, K. Klein, D.A. Flockhart, U.M. Zanger, Impact of CYP2B6 polymorphism on hepatic efavirenz metabolism in vitro. Pharmacogenomics, 8 (2007) 547-558. https://doi.org/10.2217/14622416.8.6.547

[18] C. Xu, E.T. Ogburn, Y. Guo, Z. Desta, Effects of the CYP2B6* 6 allele on catalytic properties and inhibition of CYP2B6 in vitro: implication for the mechanism of reduced efavirenz metabolism and 
other CYP2B6 substrates in vivo. Drug Metab. Dispos., 40 (2012) 717-725. https://doi.org/10.1124/dmd.111.042416

[19] H. Zhang, C. Sridar, C. Kenaan, H. Amunugama, D.P. Ballou, P.F. Hollenberg, Polymorphic variants of Cytochrome P450 2B6 (CYP2B6.4-CYP2B6.9) exhibit altered rates of metabolism for bupropion and efavirenz: a charge-reversal mutation in the K139E variant (CYP2B6.8) impairs formation of a functional cytochrome P450-reductase complex. J. Pharmacol. Exp. Ther., 338 (2011) 803-809. https://doi.org/10.1124/jpet.111.183111 


\section{Figure Legends}

Fig. 1. Quality assessment of targeted assay performance on a pooled sample (50 livers). Technical and analytical variability (A), intra-day/inter-day reproducibility (B) and linearity (C) of the assay were assessed. In panel $\mathrm{C}$, the blue shaded area represents the range of analyzed microsomal protein in the 24 samples.

A

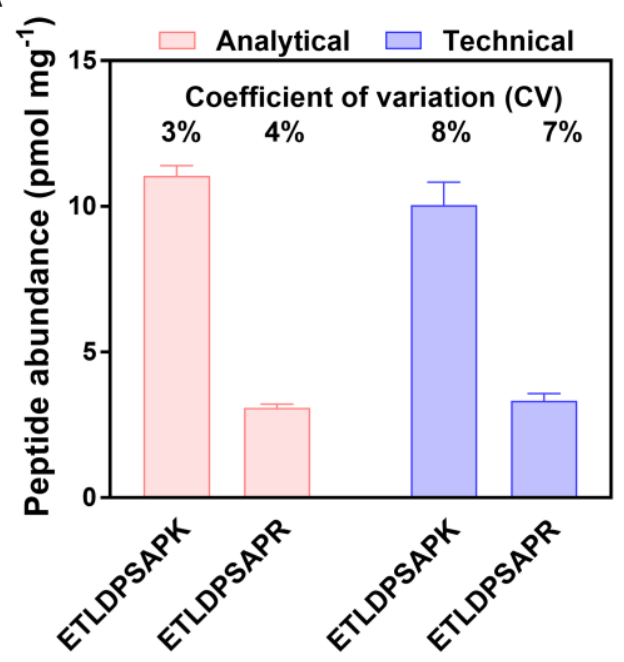

B

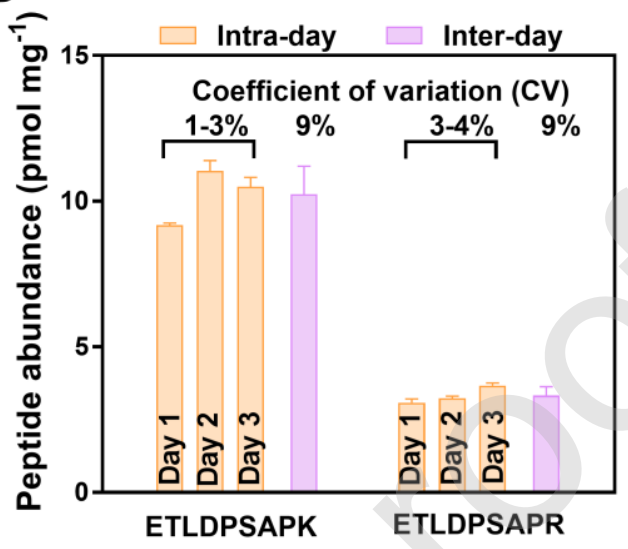

C

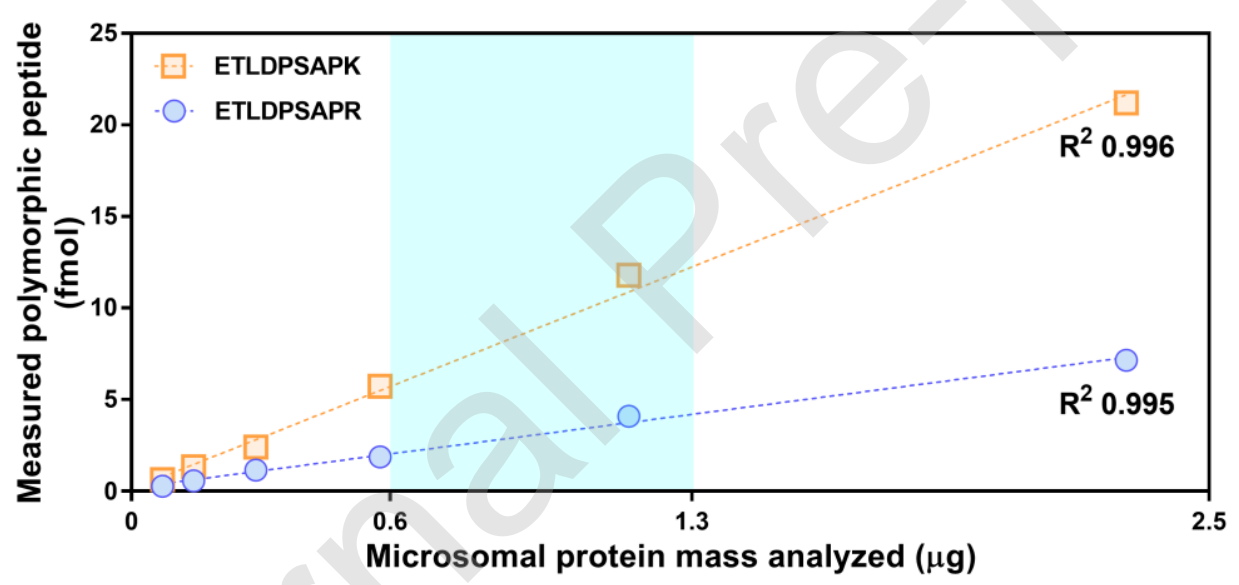

Fig. 2. Allele-specific determination of CYP2B6 using peptide standards specific to allelic variants. Peptide traces from a heterozygous $(* 1 / * 6)$ and a homozygous (wild type, $* 1 / * 1$ ) sample showing peptide signal ratios of wild type and variant analyte to QconCAT standard peptides (A). Allele-specific characterization of 21 genotyped liver samples assessed as a percentage of variant peptide signal relative to total signal; the blue shaded area represents $50 \% \pm 5 \%$ (range: $45 \%-55 \%$ ) (B). In panel A, continuous lines represent wild type peptide trace and dashed lines represent variant peptide trace. Red traces represents analyte peptide and blue traces represents the QconCAT standard peptide. In panel B, samples were either heterozygous (wild type/variant), homozygous (wild type), heterozygous expressing wild type and variant peptides (* symbol, genotype $* 4 / * 5$ in this case), heterozygous 
expressing only wild type peptide (\# symbol, genotype $* 1 / * 5$ in this case) or homozygous variant ( symbol, genotype $* 6 / * 6$ in this case). Abbreviations: $\mathrm{R}$, ratio; var, variant; wt, wild type.
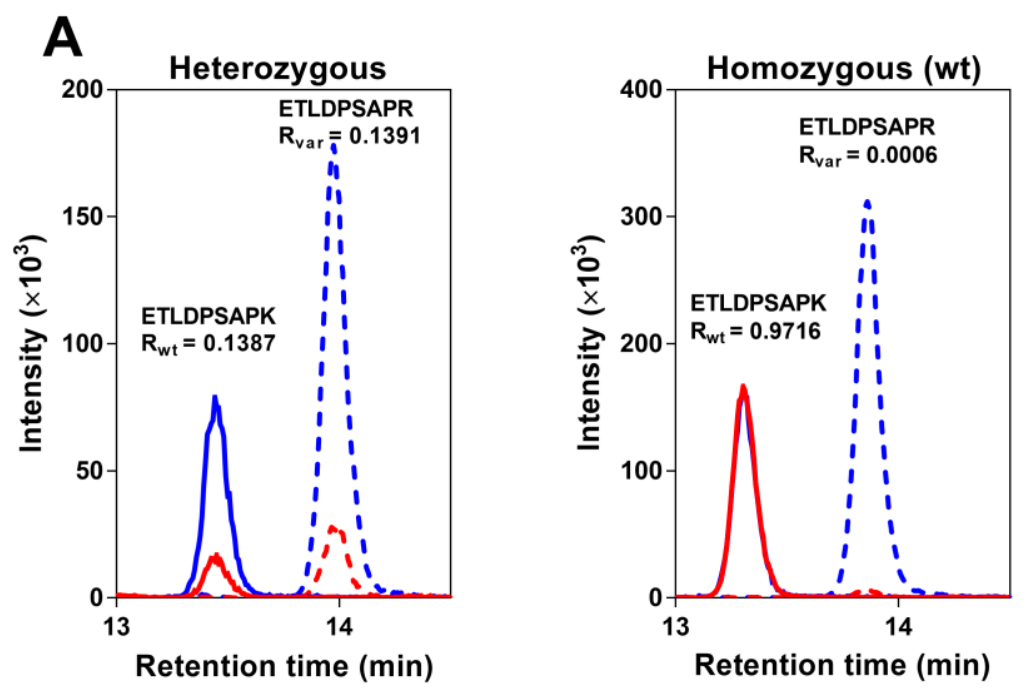

B

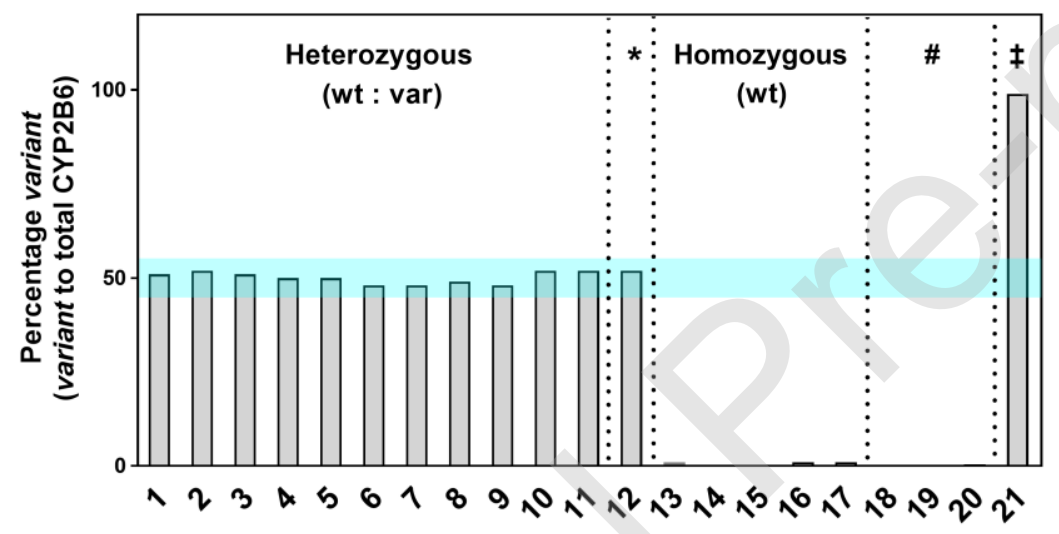

Fig. 3. Assessment of CYP2B6 abundance measured using quantitative allele-specific proteomics (QASP). The abundance of the two peptides measured using allele-specific standards (A) reflected higher average abundance with the wild type relative to the variant peptide (bars at 5.39 and $2.05 \mathrm{pmol} \mathrm{mg}^{-1}$ HLM protein, respectively). The total abundance of CYP2B6 was calculated based on measured peptide concentrations, and CYP2B6 abundance contribution to total hepatic CYP pool was assessed (B). Correlation between activity of CYP2B6 and abundance measured using the allele-specific method was tested (C) and $\mathrm{k}_{\text {cat }}$ was measured (D). Assessment of the distribution of abundance and activity data across various genotype groups (E) and comparison of protein levels and activity rates of CYP2B6 by genotype showed limited differences and large overlap between groups expressing at least one wild type allele $(\mathrm{F})$. Activity is expressed in nmol metabolite per minute per $\mathrm{mg}$ of microsomal protein; abundance is expressed in pmol enzyme per mg microsomal protein. In panel A, gray lines connect the abundance of alleles measured in the same samples. In panel B, whiskers represent CYP2B6 abundance range, the 
box represents the $25^{\text {th }}$ and $75^{\text {th }}$ percentiles, the line is the median and + symbol is the mean. In panels $\mathrm{E}$ and $\mathrm{F}$, the number of samples reflects only the genotyped samples, whereas phenotype measurements in the rest of the panels were done in all 24 samples. Abbreviations: Q-ASP, quantitative allele-specific proteomics; Rs, Spearman correlation coefficient; var, variant; wt, wild type.



\section{Table}

Table 1 Summary of proteomic multiple reaction monitoring (MRM) methods used for allele-specific measurement of CYP2B6 in human liver samples

\begin{tabular}{|c|c|c|c|c|c|}
\hline Methodology & Standard & Surrogate peptides $^{b}$ & $\begin{array}{l}\text { Peptide } m / z \\
\text { (light } / \text { heavy) }^{e}\end{array}$ & $\begin{array}{l}{\left[{ }^{13} \mathrm{C}_{6}\right] \mathrm{K} / \mathrm{R}} \\
\text { isotope } \\
\text { labeling } \\
\text { efficiency }\end{array}$ & $\begin{array}{l}\text { Monitored fragments } \\
(\mathrm{m} / z \text { light/heavy })^{e, f}\end{array}$ \\
\hline \multirow{3}{*}{$\begin{array}{l}\text { LC-MS/MS } \\
\text { proteomics } \\
\text { (multiple reaction } \\
\text { monitoring, } \\
\text { MRM) }\end{array}$} & \multirow[t]{3}{*}{$\begin{array}{l}\text { QconCAT } \\
\left(\text { MetCAT) }^{a}\right.\end{array}$} & ETLDPSAPK $^{c}$ & $479.25^{++} / 482.26^{++}$ & $95 \%$ & $\begin{array}{l}y 5\left(499.29^{+} / 505.31^{+}\right) \\
y 6\left(614.31^{+} / 620.33^{+}\right) \\
y 7\left(727.40^{+} / 733.42^{+}\right)\end{array}$ \\
\hline & & ETLDPSAPR $^{c}$ & $493.25^{++} / 496.26^{++}$ & $98 \%$ & $\begin{array}{l}y 5\left(527.29^{+} / 533.31^{+}\right) \\
y 6\left(642.32^{+} / 648.34^{+}\right) \\
y 7\left(755.40^{+} / 761.42^{+}\right)\end{array}$ \\
\hline & & GVNDNEEGFFSAR $^{d}$ & $721.32^{++} / 724.33^{++}$ & $98 \%$ & $\begin{array}{l}y 7\left(813.39^{+} / 819.41^{+}\right) \\
y 8\left(942.43^{+} / 948.45^{+}\right) \\
y 9\left(1056.47^{+} / 1062.49^{+}\right)\end{array}$ \\
\hline
\end{tabular}

${ }^{a}$ MetCAT is a QconCAT for the quantification of liver enzymes designed as previously reported [8]

${ }^{b}$ Peptide sequences contain ${ }^{13} \mathrm{C}$ isotope labeled arginine (R) or lysine (K)

${ }^{c}$ Peptides used for allele-specific quantification of CYP2B6

${ }^{d}$ The standard peptide (modified Glu-fibrinopeptide B; GVNDNEEGFFSAR) at a known concentration was used to quantify the MetCAT

${ }^{e}$ The numbers indicate mass-to-charge ratios $(\mathrm{m} / \mathrm{z}) ;+$ and ++ indicate the charge state

${ }^{f}$ Only $y$-ions were monitored to ensure the standard peptide fragments contain the labeled amino acids 
Table 2 CYP2B6 genotype in 24 liver samples and its reflection on the determined protein phenotype for the targeted non-synonymous genetic polymorphism (K262R)

\begin{tabular}{|c|c|c|c|}
\hline Liver & Genotype & $\%$ variant peptide & Comment \\
\hline 1 & $* 1 / * 6$ & $51 \%$ & Heterozygous; phenotype reflects genotype \\
\hline 2 & $* 1 / * 6$ & $52 \%$ & Heterozygous; phenotype reflects genotype \\
\hline 3 & $* 1 / * 6$ & $51 \%$ & Heterozygous; phenotype reflects genotype \\
\hline 4 & $* 1 / * 7$ & $50 \%$ & Heterozygous; phenotype reflects genotype \\
\hline 5 & $* 1 / * 7$ & $50 \%$ & Heterozygous; phenotype reflects genotype \\
\hline 6 & $* 1 / * 7$ & $48 \%$ & Heterozygous; phenotype reflects genotype \\
\hline 7 & $* 1 / * 6$ & $48 \%$ & Heterozygous; phenotype reflects genotype \\
\hline 8 & $* 1 / * 6$ & $49 \%$ & Heterozygous; phenotype reflects genotype \\
\hline 9 & $* 1 / * 7$ & $48 \%$ & Heterozygous; phenotype reflects genotype \\
\hline 10 & $* 1 / * 6$ & $52 \%$ & Heterozygous; phenotype reflects genotype \\
\hline 11 & $* 1 / * 4$ & $52 \%$ & Heterozygous; phenotype reflects genotype \\
\hline 12 & $* 4 / * 5$ & $52 \%$ & Heterozygous; phenotype reflects genotype \\
\hline 13 & $* 1 / * 1$ & $1 \%$ & Homozygous (wt); phenotype reflects genotype \\
\hline 14 & $* 1 / * 1$ & $0.10 \%$ & Homozygous (wt); phenotype reflects genotype \\
\hline 15 & $* 1 / * 1$ & $0.10 \%$ & Homozygous (wt); phenotype reflects genotype \\
\hline 16 & $* 1 / * 1$ & $1 \%$ & Homozygous (wt); phenotype reflects genotype \\
\hline 17 & $* 1 / * 1$ & $1 \%$ & Homozygous (wt); phenotype reflects genotype \\
\hline 18 & $* 1 / * 5$ & $0.10 \%$ & Heterozygous; both alleles express ETLDPSAPK \\
\hline 19 & $* 1 / * 5$ & $0.10 \%$ & Heterozygous; both alleles express ETLDPSAPK \\
\hline 20 & $* 1 / * 5$ & $0.40 \%$ & Heterozygous; both alleles express ETLDPSAPK \\
\hline 21 & $* 6 / * 6$ & $99 \%$ & Homozygous (variant); phenotype reflects genotype \\
\hline 22 & Undetermined $^{a}$ & $2 \%$ & Expected to be homozygous (wt) \\
\hline 23 & Undetermined $^{a}$ & $2 \%$ & Expected to be homozygous (wt) \\
\hline 24 & Undetermined $^{a}$ & $50 \%$ & Expected to be heterozygous (wt/variant) \\
\hline Pool & Not applicable & $34 \%$ & Pool of 50 livers \\
\hline
\end{tabular}

wt, wild type (CYP2B6*1 allele); the allele CYP2B6*5 also expresses the wild type sequence ETLDPSAPK Variant, genetic polymorphic variant (either CYP2B6*4, 6, 7, 13, 16, 19, 20, 26, 34, 36, 37 or 38) Percentages are determined from the measured peptide concentrations as $100 \times[$ ETLDPSAPR] / ([ETLDPSAPR] + [ETLDPSAPK])

${ }^{a}$ Cases where genotype data were not available and predictions were made based on proteomic data 
Table 3 Assessment of abundance and activity in different CYP2B6 genotype groups. Abundance was measured using quantitative allele-specific proteomics (Q-ASP). The results show a mirror-image trend between abundance and activity across genotypes

\begin{tabular}{|c|c|c|c|c|c|c|}
\hline $\begin{array}{l}\text { CYP2B6 } \\
\text { genotype }\end{array}$ & $\begin{array}{l}\text { Predicted } \\
\text { enzymatic } \\
\text { activity for } \\
\text { genotype }\end{array}$ & $n$ & $\begin{array}{l}\text { Q-ASP } \\
\text { Abundance } \\
(\text { pmol mg-1) }\end{array}$ & $\begin{array}{l}\text { Activity } \\
\left(\text { nmol } \text { min }^{-1}\right. \\
\text { mg-1) }^{-1}\end{array}$ & $\begin{array}{l}\mathbf{k}_{\text {cat }} \\
\left(\min ^{-1}\right)^{c}\end{array}$ & Statistical analysis \\
\hline$* 1 / * 1$ & Normal & 5 & $10.69 \pm 7.11$ & $0.070 \pm 0.064$ & $5.6 \pm 2.0$ & ANOVA \\
\hline$* 1 / * 4$ & - & 1 & 2.77 & 0.022 & 7.9 & No difference $(p>0.05)$ \\
\hline$* 1 / * 5$ & - & 3 & $7.63 \pm 10.90$ & $0.054 \pm 0.075$ & $6.9 \pm 4.3$ & \\
\hline$* 1 / * 6$ & - & 6 & $10.25 \pm 11.78$ & $0.079 \pm 0.116$ & $6.5 \pm 1.7$ & Post-hoc analysis ${ }^{d}$ \\
\hline$* 1 / * 7$ & - & 4 & $5.11 \pm 2.29$ & $0.026 \pm 0.011$ & $5.6 \pm 2.6$ & No difference $[* 1 / * 1$, \\
\hline$* 4 / * 5$ & - & 1 & 6.89 & 0.042 & 6.1 & $* 1 / * 5, * 1 / * 6, * 1 / * 7]$ \\
\hline$* 6 / * 6$ & Reduced $^{b}$ & 1 & 0.95 & 0.020 & 21.1 & $(p>0.02)$ \\
\hline
\end{tabular}

${ }^{a}$ Considered based on information from the Pharmacogene Variation Consortium database (last accessed: 14/06/2019)

${ }^{b}$ Significant effect of the genotype on activity is predicted and reflected by abundance level

${ }^{c} \mathrm{k}_{\text {cat }}$ (drug turnover number) was calculated for all samples based on Vmax and CYP2B6 abundance data

${ }^{d}$ Post-hoc analysis was carried out for sub-sets with $\mathrm{n} \geq 3$ samples using Tukey-Kramer multiple comparisons test and a Bonferonnicorrected cut-off $p$-value $\left(\alpha^{\prime}=0.017\right)$ 